\title{
Ethylene Glycol from Lignocellulosic Biomass: Impact of Lignin on Catalytic Hydrogenolysis
}

\author{
T.D.J. te Molder, S.R.A. Kersten, J. P. Lange, and M. P. Ruiz*
}

Cite This: Ind. Eng. Chem. Res. 2021, 60, 7043-7049

Read Online

ABSTRACT: Short polyols, such as ethylene glycol (EG), are a popular target of catalytic hydrogenolysis of saccharides. However, studies on the use of untreated or pretreated lignocellulosic biomass as feedstock for polyol production are scarce. In this work, we have studied the impact of lignin on the catalytic hydrogenolysis of different biomass samples, targeting ethylene glycol. We first developed a hydrogenolysis protocol that is sensitive to lignin and feedstock impurities, such as ash and extractives. A matrix of biomass feedstocks with varying lignin content has been evaluated, by subjecting poplar, pine, and hay to solvent-based (water/ethanol/acetic acid) pretreatments and by preparing physical mixtures of pure microcrystalline cellulose with organosolv
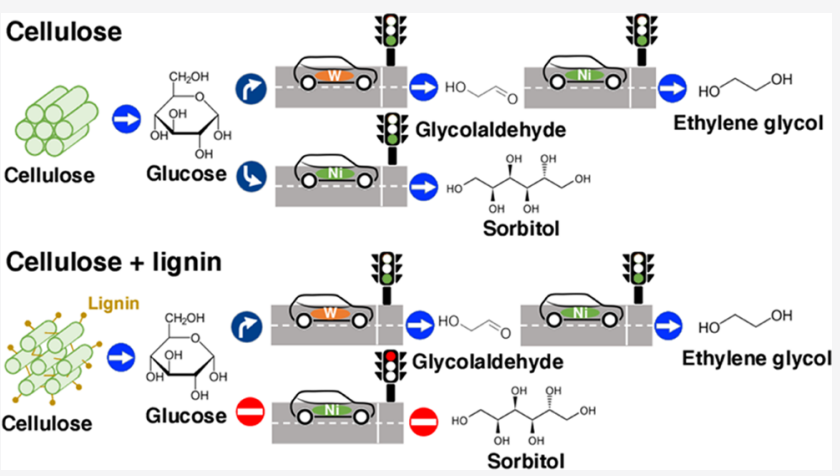
lignin. Lignin appeared to inhibit the activity of the hydrogenation catalyst, Raney-Ni, by hindering the formation of sugar alcohols in the presence as well as in the absence of the tungstate catalyst. However, lignin is not the root cause for the low EG yield typically obtained with untreated lignocellulose, as treated lignocellulose delivered high EG yields (30-35 wt \%), irrespective of the lignin concentrations, which varied between 0 and 44 wt \%, under identical demanding experimental conditions.

\section{INTRODUCTION}

The current benchmark for selective bio-ethylene glycol production is a multistep process that proceeds via fermentation of sugar to ethanol followed by dehydration to ethylene, ${ }^{1}$ which is subsequently converted to ethylene glycol (EG) via the traditional petrochemical route. ${ }^{2}$ A more direct route, but unfortunately unselective, is by hydrogenolysis of sugars, which leads to a mixture of EG, propylene glycol (PG), and glycerol., These polyols have been a popular target product from sugar because of their favorable match in atomic composition. ${ }^{3}$ The seminal paper by Zhang et al. ${ }^{5}$ demonstrated that the selectivity toward EG can be boosted by the use of a tungsten-based bifunctional catalyst. This new route has received significant attention from academia ${ }^{6}$ and industry since then.

Cellulose is the most abundant saccharide available, at modest cost, and therefore an interesting feedstock for hydrogenolysis to EG. Various authors have reported the successful conversion of microcrystalline cellulose to EG with yields as high as $\sim 75 \mathrm{wt} \%$. $^{7-9}$ Typically, the conversion is performed in hot compressed water at $245{ }^{\circ} \mathrm{C}$, ${ }^{10}$ often supplemented with an acid, ${ }^{11}$ which facilitates in situ depolymerization of cellulose to glucose. ${ }^{12}$ After hydrolysis, glucose undergoes retro-aldol condensation to glycolaldehyde, catalyzed by a homogenous tungstate (W)-species, and is subsequently hydrogenated to EG over a metal catalyst, ${ }^{12,13}$ see Figure 1. Propylene glycol (PG) is produced via a similar

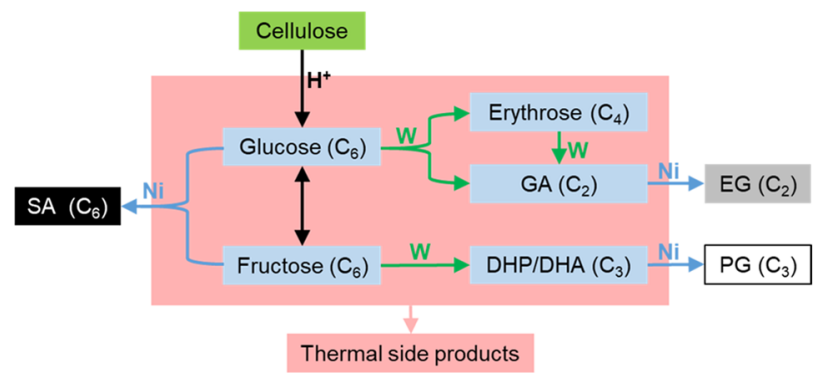

Figure 1. Simplified reaction system. EG, ethylene glycol; PG, propylene glycol; SA, sugar alcohols; DHP, dihydroxypropanal; DHA, dihydroxyacetone; GA, glycolaldehyde; $\mathrm{Ni}$, Raney-Ni catalyst; and W, homogenous tungstate catalyst.

route but is derived from fructose, which is formed by isomerization of glucose. The production of these glycols (EG and PG) competes with thermal side reactions (e.g., 5hydroxymethyl furfural (HMF) and humin formation) and the

Received: March 18, 2021

Revised: April 22, 2021

Accepted: April 27, 2021

Published: May 6, 2021 
direct hydrogenation of (mono)-saccharides to sugar alcohols (SA). A proper balance between hydrogenation and retro-aldol condensation activity is required to yield EG. ${ }^{14}$ This balance shifts to SA production in case the $\mathrm{W}$-catalyst deactivates and to thermal side products when the hydrogenation catalyst deactivates.

However, native cellulose is usually present in a matrix made of lignin ( 15-30 wt \% dry basis) and hemicellulose, in a form called lignocellulose. Understanding the impact of lignin on catalytic hydrogenolysis is key for the development of a suitable pretreatment technology that enables subsequent metal-based catalyzed valorization of the saccharide fraction. Lignin could impact the catalytic conversion to EG in several ways. For example, it may shield the cellulose, thus making it less accessible ${ }^{15}$ for further conversion, or lignin may impact the activity of the catalytic system (Figure 1). Hence, several authors explored the potential of pretreatments to free the feedstock from potentially undesired lignin. We carefully analyzed these past studies ${ }^{16-20}$ (see Supporting Information S3) and recognized that several studies underestimated their glycol yield at high lignin content because they reported it on biomass intake rather than saccharide intake. ${ }^{16,17,19}$ After expressing the EG yields on holocellulose intake basis, some data sets still showed an inverse relationship between the lignin content of the feed and EG yields ${ }^{16,17}$ (see open symbols in Figure 2). However, data from other studies, e.g., by Li et al., ${ }^{20}$

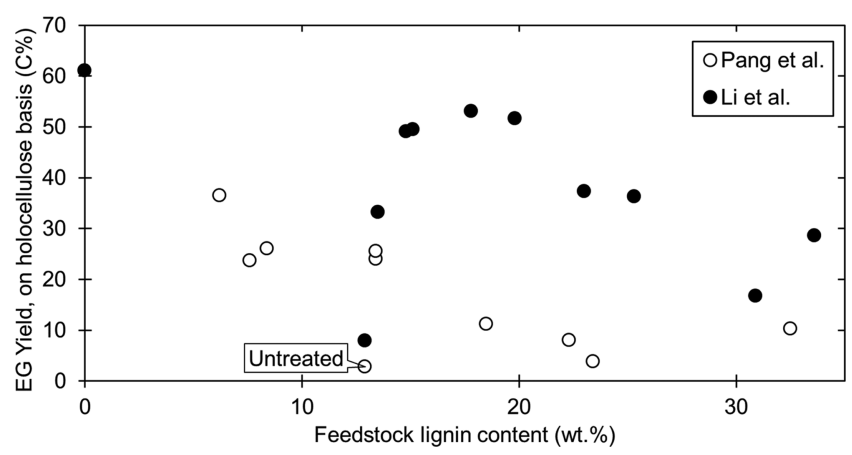

Figure 2. EG yield as a function of the feedstock lignin content, based on the hydrogenolysis data of untreated and pretreated Corn stalk from Pang et al. ${ }^{16}$ (open symbols) and different untreated feedstocks from $\mathrm{Li}$ et al. ${ }^{20}$ (closed symbols). Note: EG yields for the study by Pang et al. $^{16}$ were recalculated on holocellulose basis instead of biomass basis as reported in the original work, see Supporting Information S4 for details.

show no clear relationship between the EG yield and the lignin content of different untreated feedstocks (see closed symbols in Figure 2).

Obviously, there is no clarity yet on the role of lignin in the catalytic hydrogenolysis to EG. In this context, the aim of this study is to elucidate the role of lignin on this reaction and investigate if lignin could be the cause of possible catalyst deactivation. To do that, the hydrogenolysis conditions were selected to maximize the sensitivity of hydrogenolysis to lignin, rather than maximizing the EG yield. Three lignocellulosic biomass archetypes, namely, hay (herbaceous), poplar (hardwood), and pine (softwood) were selected, to rule out feedstock as the key variable. Moreover, different solventbased (water/ethanol/acetic acid) pretreatments were applied to these feedstocks to create samples with a wide varying residual lignin content (0-44 wt \%). Additionally, physical mixtures of pure Avicel cellulose and organosolv lignin have also been evaluated. Furthermore, we have studied the impact of lignin on the hydrogenation catalyst alone, by conducting tungstate-free experiments.

\section{MATERIALS AND METHODS}

2.1. Materials. Glacial acetic acid ( $\geq 99 \%)$, ethanol ( $\geq 99 \%$ ), Raney-Ni (W.R. Grace and Co. Raney 2800) hereafter referred to as $\mathrm{Ni}$-catalyst, sodium polytungstate ( $\geq 85 \% \mathrm{WO}_{3}$ basis) hereafter referred to as $\mathrm{W}$-catalyst, sodium hydroxide $(\geq 99 \%)$, barium carbonate $(\geq 99 \%)$, and microcrystalline cellulose (Avicel $\mathrm{PH}-101$, particle size $\sim 50 \mu \mathrm{m}$ ), hereafter referred to as cellulose, were purchased from SigmaAldrich. Avicel is a high purity cellulose, free from contaminants such as lignin, ash, and extractives. Organosolv lignin was kindly provided by ECN. Poplar wood was kindly provided by a local wooden shoe company. Pine wood (Lignocel 9) was obtained from Rettenmaier \& Söhne GmbH. Hay was purchased from a local garden and animal store. Poplar and pine of $1-2 \mathrm{~mm}$ particle size were obtained by sieving and $<53 \mu \mathrm{m}$ particle size was obtained by grinding (hammer mill) and subsequent sieving. Hay with a particle size between 53 and $355 \mu \mathrm{m}$ and smaller than $53 \mu \mathrm{m}$ particle size was obtained by grinding (hammer mill) and subsequent sieving. Biomass with a particle size smaller than $53 \mu \mathrm{m}$ was used for hydrogenolysis experiments. Pine and poplar of 1-2 $\mathrm{mm}$ and hay $(53-355 \mu \mathrm{m})$ were used for pretreatment experiments. The composition of these biomass species is reported in Table 1.

Table 1. Biomass Composition on Dry Basis (Saccharide Content, Lignin, Extractives, $o$-Acetyl-Group Determination $n=2$, Ash Determination $n=4)$ Determined for $1-2 \mathrm{~mm}$ Pine and Poplar and 53-355 $\mu \mathrm{m}$ Hay

\begin{tabular}{llrl} 
& poplar & pine & \multicolumn{1}{c}{ hay } \\
$\mathrm{H}_{2} \mathrm{O}$ extractive (wt \%) & $7.2 \pm 0.3$ & $9.2 \pm 0.4$ & $23.1 \pm 0.6$ \\
EtOH extractive (wt \%) & $1.8 \pm 0.2$ & $0.7 \pm 0.5$ & $2.0 \pm 0.4$ \\
lignin (wt \%) & $21.4 \pm 0.1$ & $24.8 \pm 0.2$ & $18.5 \pm 0.4$ \\
ash (wt \%) & $0.8 \pm 0.0$ & $0.6 \pm 0.1$ & $4.5 \pm 0.1$ \\
o-acetyl groups (wt \%) & $3.1 \pm 0.1$ & $1.4 \pm 0.1$ & $2.0 \pm 0.1$ \\
saccharide composition & & & \\
$\quad$ glucan (wt \%) & $38.7 \pm 0.1$ & $36.6 \pm 0.3$ & $22.2 \pm 0.5$ \\
$\quad$ mannan (wt \%) & $2.6 \pm 0.6$ & $10.5 \pm 0.1$ & 0 \\
$\quad$ galactan (wt \%) & 0 & $1.4 \pm 0.0$ & 0 \\
$\quad$ xylan (wt \%) & $10.9 \pm 0.2$ & $3.8 \pm 0.0$ & $10.6 \pm 0.6$ \\
arabinan (wt \%) & $0.5 \pm 0.2$ & $1.4 \pm 0.1$ & $2.9 \pm 0.1$ \\
\hline
\end{tabular}

2.2. Biomass Pretreatment. Biomass was pretreated under different conditions to obtain samples with varying lignin content. For this, we used various mixtures of water and acetic acid and ethanol as medium as (1) it allows lignin removal and (2) avoids the use of inorganic supplements that potentially affect the hydrogenolysis catalysts. The conditions, time, temperature, and solvent composition, of the pretreatment experiments, as well as the lignin content of the dried solid residue, are shown in Table 2 . The solid residues were used as feed for catalytic hydrogenolysis. It is worth noting that the samples with high lignin content, $\mathrm{J}$ and $\mathrm{K}$ in Table 2, were prepared by a pretreatment using a solvent with a high proportion of water $(>80 \%)$, targeting the selective removal of hemicellulose while retaining the lignin in the sample. 
Table 2. Lignin Content of Pretreated Samples ${ }^{a}$

$\begin{array}{clc}\text { pretreatment } & \text { feed } & \text { substrate lignin content (wt \%, dry) } \\ \text { A } & \text { poplar } & 1.5 \pm 0.0 \\ \text { B } & \text { poplar } & 3.6 \pm 0.4 \\ \text { C } & \text { poplar } & 6.2 \pm 0.4 \\ \text { D } & \text { pine } & 10.0 \pm 1.9 \\ \text { E } & \text { poplar } & 11.5 \pm 1.3 \\ \text { F } & \text { poplar } & 15.5 \pm 2.5 \\ \text { G } & \text { pine } & 19.8 \pm 2.1 \\ \text { H } & \text { hay } & 21.3 \pm 1.3 \\ \text { I* } & \text { poplar } & 24.6 \pm 0.7 \\ \text { J } & \text { poplar } & 38.7 \pm 2.3 \\ \text { K } & \text { hay } & 45.9 \pm 1.9\end{array}$

${ }^{a}$ See Supporting Information S4 and Table S16 for operational conditions and substrate saccharide composition. *Biomass loading in this experiment was $\sim 20$ wt $\%$.

The pretreatment experiments were performed in a $200 \mathrm{~mL}$ stirred autoclave. In a typical experiment, the reactor was charged with $10 \mathrm{~g}$ of biomass (oven-dried at $105^{\circ} \mathrm{C}$ ) and $90 \mathrm{~g}$ of the corresponding solvent. Air was displaced by flushing the autoclave three times with nitrogen. The pretreatment was carried out for $1-5 \mathrm{~h}$ at $180-200{ }^{\circ} \mathrm{C}$. Afterward, the solid residue was washed with a mixture of $50 / 50 \mathrm{w} / \mathrm{w}$ water/ ethanol until no color change of the liquid was observed. Subsequently, the solid residue was dried by flushing with nitrogen at room temperature until no weight change of the solid residue was observed.

2.3. Catalytic Hydrogenolysis. Catalytic hydrogenolysis experiments were carried out in a $45 \mathrm{~mL}$ batch autoclave. The reactor was mounted on a pneumatic arm. A preheated fluidized sand bed and a water bath were allowed for rapid heating and cooling, respectively. The autoclave was equipped with a hollow shaft stirrer, a pressure transducer, and a thermocouple located on the bottom of the vessel. Details about the setup can be found elsewhere. ${ }^{21}$

Typically, the autoclave was charged with $14.25 \mathrm{~g}$ of deionized water, $0.75 \mathrm{~g}$ of biomass, $0.021 \mathrm{~g}$ of retro-aldol catalyst (sodium polytungstate), $0.09 \mathrm{~g}$ of hydrogenation catalyst (Raney-Ni, dry basis), and $0.3 \mathrm{~g}$ of acetic acid together with $\mathrm{NaOH}$ to buffer the solution to $\mathrm{pH}$ 3.3. Prior to use, Raney-Ni was thoroughly washed with deionized water until the $\mathrm{pH}$ of the washing water became neutral. Raney-Ni was dosed to the autoclave as a well-homogenized slurry in water. The reactor was sealed and tested for leaks with nitrogen at $\sim 120$ bar. Afterward, the autoclave was flushed twice with nitrogen, twice with hydrogen, and then pressurized with hydrogen to $\sim 60$ bar. The stirrer was then started $(\sim 1300$ $\mathrm{rpm}$ ), and the autoclave was submerged in the fluidized sand bed. The reaction time was started once the reactor attained a temperature of $245^{\circ} \mathrm{C}$, which typically took less than $10 \mathrm{~min}$. The residence time was limited to $1 \mathrm{~h}$, as we did not observe a substantially higher EG yield (8 wt \%) for untreated poplar and lower solid residue ( $23 \mathrm{wt} \%)$ when extending the reaction time to $5 \mathrm{~h}$ ( $10 \mathrm{wt} \% \mathrm{EG}$ yield, $23 \mathrm{wt} \%$ solid residue).

After the desired reaction time, typically $1 \mathrm{~h}$, the autoclave was rapidly cooled by submerging it in the water bath. Once at room temperature, the autoclave was depressurized, opened, and the reactor content was transferred to a glass bottle. A liquid sample was taken and subjected to high-performance liquid chromatography (HPLC) analysis and $\mathrm{pH}$ measurement (Metrohm LL Unitrode Pt $1000 \mathrm{pH}$ probe details). The remaining catalyst and unconverted residue were collected in a separate bottle by flushing the reactor with deionized water. The yield was calculated according to eq 1 , in which $m_{\text {product }}$ is the mass of the product (e.g., EG), $m_{\text {feed }}$ is the mass of the biomass fed, and $f_{\text {lignin }}, f_{\mathrm{H}_{2} \mathrm{O} \text {-extract }} f_{\text {EtOH-extract }}$ and $f_{\text {ash }}$ are the lignin, water extractives, ethanol extractives, and ash weight fractions of the dry feedstock. As such, the product yield is expressed on lignin-, extractive-, $o$-acetyl group- and ash-free basis, i.e., on holocellulose basis.

$$
\begin{aligned}
& \text { yield }(\text { wt } \%)= \\
& \qquad \frac{m_{\text {product }}}{m_{\text {feed }}\left(1-f_{\text {lignin }}-f_{\mathrm{H}_{2} \mathrm{O} \text {-extract }}-f_{\text {EtOH-extract }}-f_{\text {ash }}-f_{\text {acetyl }}\right)} \times 100
\end{aligned}
$$

A proper measurement of the conversion is hindered by several factors: (1) mass losses during emptying of the autoclave, (2) inability to properly separate Raney nickel and solid residue (in particular for untreated poplar), (3) the formation of solids (e.g., humins) during catalytic hydrogenolysis, and/or (4) the presence of inconvertible material (e.g., polymerized lignin). As such, we decided to report yields and not selectivities as the error in conversion would impact the selectivity too severely.

The $\mathrm{pH}$ is known to be critical for robust operation, see Supporting Information S1.1. Besides acetic acid, other buffering agents were found less suitable, as discussed in Supporting Information S1.1. The $\mathrm{pH}$ of the liquid product obtained at the end of run was typically between 3.0 and 3.6.

2.4. Analytical Methods. 2.4.1. Biomass Characterization. The lignin content of the feedstocks was quantified in duplicate by a two-step hydrolysis with sulfuric acid. ${ }^{22}$ The biomass of the $o$-acetyl content was obtained after acetic acid quantification by HPLC analysis of the residual liquid fraction. The extractive contents of the feedstocks were quantified by Soxhlet extraction with water for $24 \mathrm{~h}$ followed by ethanol extraction according to the protocol. ${ }^{23}$ The ash content of the feedstocks was quantified by dry oxidation at $575{ }^{\circ} \mathrm{C}$ for 24 h. $^{24}$

2.4.2. High-Performance Liquid Chromatography. HPLC analysis for the quantification of hydrogenolysis products (EG, PG, SA) was performed on an Agilent 1200 series (Hi-Plex- $\mathrm{H}^{+}$ column) operated at $65{ }^{\circ} \mathrm{C}$, running with $5 \mathrm{mM} \mathrm{H}_{2} \mathrm{SO}_{4}$ as the eluent $\left(0.6 \mathrm{~mL} \mathrm{~min}{ }^{-1}\right)$, and equipped with a refractive index detector (RID) and a variable wavelength detector (VWD) operated at $285 \mathrm{~nm}$. The samples were filtered (Whatman 0.2 $\mu \mathrm{m}$ filter) prior to HPLC analysis. Samples were diluted with deionized water when necessary. Calibration curves were established for ethylene glycol (EG), propylene glycol (PG), and sorbitol, mannitol and xylitol (SA). Identification and quantification of monosaccharides (glucose, xylose, galactose, arabinose, mannose, rhamnose) were performed on the same machine (Agilent 1200 series) but operated with a Hi-Plex Pb column at $70{ }^{\circ} \mathrm{C}$ and deionized water as the eluent.

\section{RESULTS AND DISCUSSION}

3.1. Hydrogenolysis Protocol. In this work, a sensitive hydrogenolysis protocol to maximize the deactivating role of feedstock components (e.g., lignin) has been designed. In our experiments, both a soluble tungsten species (sodium polytungstate, hereafter referred to as $\mathrm{W}$-catalyst) and Raney nickel catalyst (referred to as Ni-catalyst) have been employed. Furthermore, a 2 wt \% buffered acetic acid solution has been used to control the $\mathrm{pH}$ at around $\sim 3.3$. Besides the main 
products (EG, PG, SA), numerous side products are formed in minor amounts, such as 1,2-butanediol, erythritol, glycerol, light alcohols, and light organic acids. However, for the sake of clarity, we limit the discussion to the main products, EG, PG, and SA.

We investigated the impact of $\mathrm{W}$ and $\mathrm{Ni}$ loading on product yield for biomass loading of $1 \mathrm{wt} \%$ and found little yield differences when using pure cellulose or untreated poplar, see the Supporting Information (S1.2 and S1.3). It is worth noting that changes in the structure of the biomass after pretreatment (e.g., improved cellulose accessibility) could also have an impact on the performance of the hydrogenolysis system (Figure 1). However, when operating in excess of catalyst at 1 wt $\%$ biomass loading, it was observed that both untreated poplar $(<53 \mu \mathrm{m})$ and microcrystalline cellulose $(\sim 50 \mu \mathrm{m})$ resulted in similar EG yields ( $\sim 40$ wt \%) (see the Supporting Information (Table S1, entries 1 and 2)). This means that lignin is not hindering the micro-accessibility (i.e., at the cellwall level), at least for these feeds under the conditions studied.

A similar observation was made for poplar, basswood, ash tree, and birch $(\sim 50$ wt $\%$ EG yield $)$, compared to microcrystalline cellulose (61 wt \% EG yield), in the work of $\mathrm{Li}$ et al., ${ }^{20}$ see Figure 2. Also, from the studies by Pang et al. ${ }^{17}$ and Zhou et al. ${ }^{19}$ on Miscanthus and Jerusalem artichoke, respectively, it was concluded that pretreated and untreated samples gave the same EG yield (on holocellulose basis), when operating at 1 wt \% biomass loading, see the Supporting Information (S3, Figures S7 and S11). Thus, all of these observations indicate that the biomass structure is not significantly limiting the EG yield, at least for the examples mentioned. They also suggest that differences in the lignin structure in the various feedstocks may not critically impact the catalytic hydrogenolysis. This preliminary conclusion would require further studies for confirmation.

Nevertheless, operation at high biomass loading will be imperative for commercial operation, as discussed elsewhere. ${ }^{25}$ We then raised the biomass loading to $5 \mathrm{wt} \%$ and increased the catalyst loading proportionally, as proposed in the literature, ${ }^{17}$ and found again limited difference between pure cellulose and untreated lignocellulose, see the Supporting Information (Table S1, entries 3 and 4). We then decided to increase the sensitivity of the protocol for catalyst poisoning by increasing the biomass loading without increasing the catalyst loading. With 5 wt \% biomass loading and $0.03 \mathrm{~W}$-catalyst/ biomass and $0.12 \mathrm{Ni}$ /Biomass mass ratio, we eventually achieved good differentiation, with an EG yield of $32.2( \pm 0.5)$ wt $\%(n=2)$ for microcrystalline cellulose but only $8.3( \pm 0.4)$ wt $\%(n=2)$ for untreated poplar, 8 wt $\%$ for pine, and 4 wt $\%$ for hay. Details on the development of this hydrogenolysis protocol can be found in Supporting Information S1.

3.2. W-Catalyst-Free Experiments. We hypothesized that lignin may foul the hydrogenation catalyst, thereby blocking and inhibiting the hydrogenation of intermediates (e.g., glycolaldehyde, monosaccharides), which would result in poor EG, PG, and SA yields. Similar statements have been postulated previously. ${ }^{16,17}$

To systematically study the deactivation of the hydrogenation catalyst by lignin, $\mathrm{W}$-catalyst-free experiments with physical mixtures of cellulose and organosolv lignin in varying ratios were run (see Section 2, Materials and Methods). In the absence of $\mathrm{W}$-catalyst, the dominant reaction product should be SA (see Figure 1). Indeed, SA was the main product for pure cellulose experiments, with $32 \pm 0.8$ wt $\%$ yield (performed in duplicate), but the yield dropped to $\sim 13 \mathrm{wt}$ $\%$ when cellulose was mixed with 5 wt $\%$ of lignin and decreased further at higher lignin loading, e.g., to 9 wt \% at 25 wt \% lignin (see Figure 3, open circles). Note that the product

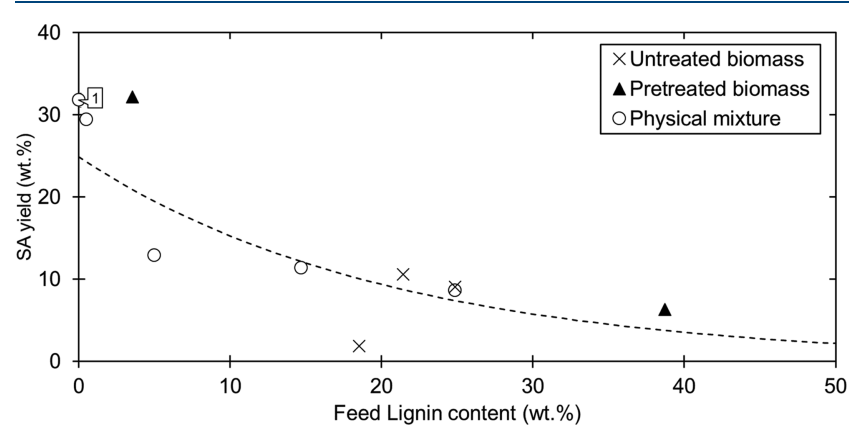

Figure 3. SA yield after hydrogenolysis over Raney nickel. Relevant conditions: $0.12 \mathrm{Ni}$-catalyst to biomass ratio, biomass loading $=5 \mathrm{wt}$ $\%, T=245{ }^{\circ} \mathrm{C}, t=1 \mathrm{~h}, P_{\mathrm{H}_{2}}$ (initial) $=60$ bar, and $\mathrm{pH}_{\text {initial }}=\sim 3.3$. Experiment 1 was performed in duplicate; the error $( \pm 0.8$ wt \%) bar is too small to be observed. Lines are drawn for clarity.

yields are not limited by incomplete cellulose conversion as a catalyst-free experiment gave a cellulose conversion $>90 \mathrm{wt} \%$ after $1 \mathrm{~h}$. Data for untreated poplar and pine appeared on the same line, as did a pretreated poplar sample with 39 wt \% lignin (see Figure 3, crosses and closed triangle). A pretreated sample with $3.6 \mathrm{wt} \%$ lignin showed a higher SA yield than expected. In contrast, untreated hay fell well below the line with only 2 wt \% of SA (see Figure 3, cross).

This leads to the following conclusions. First, lignin might deactivate the hydrogenation catalyst. This statement is further supported by a correlation coefficient of -0.85 between the SA yield and feed lignin content. Second, this deactivation appears to be similar for the native lignin found in untreated feedstock and the processed lignin found in pretreated feed and used for cellulose/lignin mixtures, which suggests that the celluloselignin interaction does not significantly affect the hydrogenolysis outcome under the conditions studied. Third, there seem to be other deactivating elements than lignin, as indicated by the hay experiments. This point warrants further studies.

Figure 3 is also conveying a worrisome message. The Nicatalyst appears to lose $\sim 50 \%$ of its initial activity after having seen about half its weight of lignin. However, industrial processes are reported to normally operate with an overall catalyst consumption of $1 \mathrm{~kg}$ per ton of the product. ${ }^{25}$ Unless the Ni-catalyst activity stabilizes after initial poisoning with lignin, such consumption target would then limit the lignin content of the feed to the impractical level of some $<0.1 \mathrm{wt} \%$ or would require the $\mathrm{Ni}$-catalyst to be regenerable. Both options clearly need further studies.

3.3. W-Catalyst + Ni-Catalyst. Then, pretreated biomass samples with varying lignin contents and physical mixtures of cellulose and lignin in the presence of $\mathrm{W}$-catalyst and $\mathrm{Ni}$ catalyst were tested, to study the overall deactivating role of lignin on EG yield according to our hydrogenolysis protocol ( 5 wt \% biomass loading, $0.12 \mathrm{Ni}$-catalyst to biomass ratio, and $0.03 \mathrm{~W}$-catalyst to biomass ratio).

Hydrogenolysis experiments are labor intensive; therefore, three key data points were selected and run in duplicate to validate the reproducibility of our protocol and labeled in Figure 4, as 1,2, and 3. The error of these experiments is small; that is, the error bars are typically too small to be observed and 

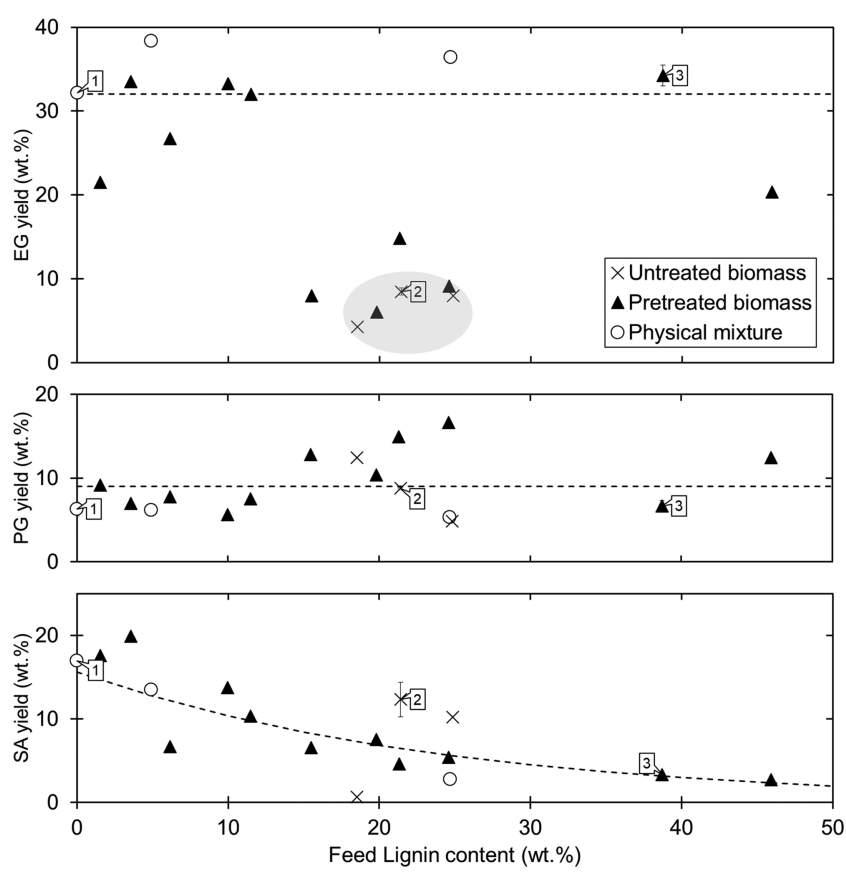

Figure 4. Product yields (EG, PG, and SA) as a function of the feedstock lignin content in the catalytic hydrogenolysis. Reaction conditions: 5 wt $\%$ biomass loading, $T=245^{\circ} \mathrm{C}, t=1 \mathrm{~h}, P_{\mathrm{H}_{2}}$ (initial) $=60$ bar, $\mathrm{pH}_{\text {initial }}=\sim 3.3$, Ni-catalyst to biomass mass ratio 0.12 , and $\mathrm{W}$-catalyst to biomass mass ratio 0.03 . Experiments 1,2 , and 3 were performed in duplicate; the error bars are typically too small to be observed. Lines and gray area are drawn for clarity. A more detailed figure is provided in the Supporting Information (Figure S6).

the difference in EG yield is less than \pm 1 wt \%. This shows that our measurements are reproducible and, therefore, reliable.

When conducting experiments under our sensitive test conditions, it was confirmed that untreated biomass delivered much lower EG yield, namely, 4-8 wt \% (see crosses in the gray area in Figure 4), instead of 32 wt \% for cellulose. It was found that the EG yield for pretreated biomass was either very similar to the untreated biomass, namely, $\leq 10 \mathrm{wt} \%$, or similar to pure cellulose at $\sim 32 \mathrm{wt} \%$ (see filled triangles in Figure 4). Thus, no plausible relation with the feed lignin content was observed (correlation coefficient of -0.05 , see Supporting Information S3.6). In fact, very high EG yield, 35 wt \%, was obtained for a sample with an extremely high lignin content (39 wt \%). Moreover, the addition of organosolv lignin to cellulose as a physical mixture also delivered similarly high EG yields, irrespective of the lignin content. This also suggests that the precise location of lignin, i.e., bulk or surface, may not be critical, but this hypothesis requires further studies. These results suggest that the beneficial effect of most pretreatments on the EG yield is not so much due to the removal of lignin but due to other changes in the biomass. The topic is a subject of further investigation in our laboratory.

We realized here that the effective catalyst to holocellulose ratio is increasing (by $\sim 30 \%$ ) as lignin content of the feed is reduced from 25 to 0 wt \%, and this could affect the EG yield. But this possibility could be discarded by an additional test with constant holocellulose content, instead of constant biomass content, see the Supporting Information (S2.1, Figure S5).

The lignin content of pretreated samples and physical mixture of cellulose and lignin did not seem to affect the PG yield (Figure 4). However, it depressed the competitive hydrogenation of sugar to SA (correlation coefficient of $-0.65)$, as was also previously observed for W-catalyst-free experiments (see Figure 3). Here, untreated poplar and pine fell outside this general trend by delivering higher SA yield than treated samples or physical mixtures of similar lignin content (see Figure 4). The SA yield of $\sim 12$ wt $\%$ is fairly similar as for W-catalyst-free experiments, namely, $\sim 10 \mathrm{wt} \%$ (see Figure 3), while the EG yields were very poor ( $8 \mathrm{wt} \%$ ). All of these suggest that the $\mathrm{W}$-catalyst was readily deactivated.

Lignin inhibits the hydrogenation of saccharides over Raney$\mathrm{Ni}$ to SA but retains sufficient activity to hydrogenate EG and PG intermediates that are notoriously more reactive. ${ }^{26}$ Apparently, hydrogenation of GA to EG is not rate limiting under the current concentrations and conditions, even at such low Ni-catalyst/biomass ratio of 0.12 . Moreover, it has been observed that lignin does not significantly affect the retro-aldol condensation reaction. Most importantly, lignin is not the root cause for the low EG yield after hydrogenolysis of untreated lignocellulosic biomass. The true root cause should be sought elsewhere and is currently under further investigation.

3.4. Comparison with Previous Studies. These observations contradict earlier studies ${ }^{16,17}$ claiming that lignin is a key inhibitor in the catalytic hydrogenolysis to EG. An overview of relevant literature studies is presented in Table 3. Also, a statistical analysis of the literature data and results of this work was performed. Several studies reported their glycol yield on biomass intake ${ }^{16,17,19}$ and were recalculated on holocellulose intake for the present discussion. Consequently, the reported depression of EG yield by lignin vanishes for pretreated Miscanthus (Pang et al. ${ }^{17}$ ) and pretreated Jerusalem artichoke (Zhou et al. ${ }^{19}$ ), two herbaceous types of biomass, when running at 1 wt \% biomass loading, see the Supporting Information (S3, Figures S7 and S11).

A key data set has been provided by $\mathrm{Li}$ et al., ${ }^{20}$ who performed hydrogenolysis of 10 untreated lignocellulose species over $\mathrm{Ni}-\mathrm{W}_{2} \mathrm{C}$ catalysts at 1 wt \% biomass loading.

Table 3. Studies That Investigated the Catalytic Hydrogenolysis of (Pretreated) Biomass Targeting EG and PG

\begin{tabular}{|c|c|c|c|c|c|}
\hline refs & feedstock & catalyst & $\begin{array}{l}\text { biomass loading } \\
\text { (wt \%) }\end{array}$ & $\begin{array}{l}\text { W-catalyst/biomass } \\
\text { (w/w) }\end{array}$ & $\begin{array}{l}\text { hydrogenation catalyst/biomass } \\
\qquad(\mathrm{w} / \mathrm{w})\end{array}$ \\
\hline 16 & (pretreated)-corn stalk & $2 \% \mathrm{Ni}-\mathrm{W}_{2} \mathrm{C}$ & 1 & & $0.3^{a}$ \\
\hline 20 & various & $4 \% \mathrm{Ni}-30 \% \mathrm{~W}_{2} \mathrm{C} / \mathrm{AC}$ & 1 & & $0.4^{a}$ \\
\hline 19 & $\begin{array}{l}\text { (pretreated)-Jerusalem } \\
\text { artichoke }\end{array}$ & $\mathrm{WO}_{3}+$ Raney-Ni & 1 & 0.3 & 0.3 \\
\hline 17 & (pretreated)-miscanthus & $\mathrm{H}_{2} \mathrm{WO}_{4}+$ Raney-Ni & $1,6,10$ & 0.12 & 0.12 \\
\hline 18 & (pretreated)-barley straw & $\mathrm{Ru}-\mathrm{W} / \mathrm{AC}$ & 4.8 & & $0.1^{a}$ \\
\hline this work & (pretreated)-poplar, pine, hay & $\mathrm{SPT}+$ Raney-Ni & 5 & 0.03 & 0.12 \\
\hline
\end{tabular}

${ }^{a}$ A bifunctional catalyst was used. 
The EG yields obtained in their work versus the respective lignin content of the feedstocks are plotted in Figure 2. Interestingly, hydrogenolysis of various untreated hardwood species (poplar, basswood, ash tree, and birch) resulted in EG yields of $49 \mathrm{C} \%$ or higher, comparable to hydrogenolysis of microcrystalline cellulose $\left(Y_{\mathrm{EG}}=61 \mathrm{C} \%\right)$. There is no apparent relation between the feedstock lignin content and the EG yield, which is supported by a poor correlation coefficient of -0.26 and which we found for the data set of $\mathrm{Li}$ et al., ${ }^{20}$ see Supporting Information S3.6. In fact, the poorest EG yield, $8 \mathrm{C}$ $\%$, was obtained for corn stalk, which had the lowest lignin content, $13 \mathrm{wt} \%$, of all untreated feedstocks. In a different study, Pang et al. ${ }^{16}$ also observed a low EG yield of only $3 \mathrm{C} \%$ for untreated corn stalk. It is, therefore, unconvincing from Pang et al. $^{16}$ to label lignin as the key inhibitor for the hydrogenolysis of corn stalk. Furthermore, Pang et al. ${ }^{16}$ found much higher EG $(\sim 25 \mathrm{C} \%)$ and PG $(\sim 15 \mathrm{C} \%)$ yields for pretreated samples of similar lignin content as the untreated feed (EG $\sim 3 \mathrm{C} \%$ and $\mathrm{PG} \leq 3 \mathrm{C} \%$ ).

The studies by Fabičovicová et al. ${ }^{18}$ on Barley straw and Pang et al. ${ }^{17}$ on Miscanthus were performed at substantially higher biomass loading ( $\geq 4.8$ wt \%). In the work by Fabičovicová et al., ${ }^{18}$ the EG yield was $2 \mathrm{C} \%$ for untreated barley straw, whereas an EG yield of $24 \mathrm{C} \%$ was obtained for a pretreated sample with similar lignin content $(\sim 20 \mathrm{wt} \%)$ as the untreated barley straw. Furthermore, an optimum EG yield was not found in the samples with the lowest lignin content. Again, these data hint that lignin is not the key inhibitor.

In the work by Pang et al. ${ }^{17}$ for experiments at higher biomass loading ( $\geq 6 \mathrm{wt} \%$ ), the lignin content of the feed correlated very well with the EG yield $(-0.96)$. However, pretreatment, considered as a binary parameter, resulted in a good correlation as well (0.8). It is, therefore, not possible to ascribe the increase in EG yield solely to the feedstock lignin content. Furthermore, the lignin content range of this data set was limited, namely, from 1.4 to $16 \mathrm{wt} \%$, and no samples with higher lignin content than the untreated feed were tested. Note that our hydrogenolysis protocol was very similar to the hydrogenolysis protocol applied by Pang et al. ${ }^{17}$ on the catalytic hydrogenolysis of Miscanthus. Both studies applied the same Raney-Ni to biomass ratio (0.12). However, in our study, four times less W-catalyst was used, namely, a W-catalyst to biomass mass ratio of 0.03 , compared to 0.12 in the work by Pang et al. ${ }^{17}$

Beyond the study by $\mathrm{Li}$ et al., ${ }^{20}$ all other studies share the choice for a herbaceous type biomass (Miscanthus, ${ }^{17}$ barley straw, ${ }^{18}$ Jerusalem artichoke, ${ }^{19}$ corn stalk $\left.^{16}\right)$. These are typically richer in extractives and ash and leaner in lignin, compared to woody biomass. $^{27,28}$ In view of the literature results and this work, it can be concluded that lignin inhibits the hydrogenation function but is not the key inhibitor on the EG yield.

\section{CONCLUSIONS}

In this work, we have focused on the conversion of three lignocellulosic biomass archetypes, hay (herbaceous), poplar (hardwood) and pine (softwood), which are inexpensive and abundant feeds. We investigated the impact of lignin on the hydrogenolysis performance.

A hydrogenolysis protocol that is sensitive to lignin and feedstock impurities, such as ash and extractives, has been developed. It also allows $\mathrm{pH}$ control and decoupling of the acidic, retro-aldol condensation and hydrogenation functionalities of the hydrogenolysis system (Figure 1).

Key experiments were performed in duplicate and showed that the reproducibility of the hydrogenolysis test is very high, i.e., the EG yield difference between the duplicates was less than \pm 1 wt $\%$. Under the same experimental conditions, untreated biomass gave a poor EG yield (4-8 wt \%), whereas microcrystalline cellulose gave an EG yield of $32 \mathrm{wt} \%$, at $5 \mathrm{wt}$ $\%$ biomass loading. However, a similar EG yield ( $40 \mathrm{wt} \%)$ for poplar $(<53 \mu \mathrm{m})$ and cellulose at $1 \mathrm{wt} \%$ biomass loading was obtained when operating in excess of catalyst. This shows that the EG yield was not limited by the micro-accessibility of cellulose (at cell-wall level) in our tests, which evidences that our hydrogenolysis test is truly sensitive to feedstock impurities, e.g., lignin.

W-catalyst-free experiments showed that the hydrogenation catalyst is inhibited by lignin as the SA yield decreased from 33 to $6 \mathrm{wt} \%$ with increase in the feed lignin content. This observation holds for physical mixtures of cellulose and lignin, as well as pretreated biomass samples. Native lignin (i.e., untreated biomass) did not deviate from processed lignin. The SA yield also decreased with increasing the feed lignin content for experiments with both W-catalyst and hydrogenation catalyst present. However, the EG yield did not show an apparent relation with the feed lignin content. Blends of cellulose and lignin powder systematically gave high EG yields, $\geq 32$ wt \%. Pretreatment of biomass increased the EG yield in most cases to $\sim 32 \mathrm{wt} \%$, even for a sample with a lignin content of 39 wt \%. Hence, an important distinction should be made: lignin inhibits the hydrogenation of sugars over Raney$\mathrm{Ni}$ to SA, but Raney-Ni appears to maintain sufficient activity to hydrogenate the more reactive glycolaldehyde and glyceraldehyde to EG and PG, respectively.

Thus, lignin may deactivate the hydrogenation function but is not the root cause for the low EG yield obtained after hydrogenolysis of untreated biomass. Furthermore, the results show that the homogenous tungstate catalyst is not deactivated by lignin.

\section{ASSOCIATED CONTENT}

\section{SI Supporting Information}

The Supporting Information is available free of charge at https://pubs.acs.org/doi/10.1021/acs.iecr.1c01063.

Detailed information on the hydrogenolysis protocol; literature study and statistical analysis; supporting graphs on the results; and pretreatment conditions and substrate composition (PDF)

\section{AUTHOR INFORMATION}

\section{Corresponding Author}

M. P. Ruiz - Sustainable Process Technology, Faculty of Science and Technology, University of Twente, 7522 NB Enschede, The Netherlands; 10 orcid.org/0000-0003-14375578; Email: m.p.ruizramiro@utwente.nl

\section{Authors}

T.D.J. te Molder - Sustainable Process Technology, Faculty of Science and Technology, University of Twente, 7522 NB Enschede, The Netherlands

S.R.A. Kersten - Sustainable Process Technology, Faculty of Science and Technology, University of Twente, 7522 NB 
Enschede, The Netherlands; $\odot$ orcid.org/0000-0001-83332649

J. P. Lange - Sustainable Process Technology, Faculty of Science and Technology, University of Twente, $7522 \mathrm{NB}$ Enschede, The Netherlands; Shell Global Solutions International B.V., Shell Technology Centre Amsterdam, 1031 HW Amsterdam, The Netherlands; (i) orcid.org/00000001-6567-2957

Complete contact information is available at:

https://pubs.acs.org/10.1021/acs.iecr.1c01063

\section{Notes}

The authors declare no competing financial interest.

\section{ACKNOWLEDGMENTS}

The authors would like to thank Shell Global Solutions International B.V. for funding this research, and Benno Knaken, Johan Agterhorst, Ronald Borst, and Erna FränzelLuiten for the technical support.

\section{LIST OF ABBREVIATIONS}

EG ethylene glycol

PG propylene glycol

SA sugar alcohols (sorbitol, mannitol, xylitol)

GA glycolaldehyde

DHP dihydroxypropanal

DHA dihydroxyacetone

HMF hydroxymethylfurfural

Ni-catalyst Raney nickel

$\mathrm{W}$-catalyst $\mathrm{WO}_{x}$-based catalyst, sodium polytungstate in this work

\section{REFERENCES}

(1) Tuck, C. O.; Perez, E.; Horvath, I. T.; Sheldon, R. A.; Poliakoff, $\mathrm{M}$. Valorization of biomass: deriving more value from waste. Science 2012, 337, 695-699.

(2) Rebsdat, S.; Mayer, D. Ethylene Glycol. In Ullman's Encyclopedia of Industrial Chemistry; Wiley-VCH Verlag GmbH \& Co., 2000.

(3) Ruppert, A. M.; Weinberg, K.; Palkovits, R. Hydrogenolysis goes bio: from carbohydrates and sugar alcohols to platform chemicals. Angew. Chem., Int. Ed. 2012, 51, 2564-2601.

(4) Marchesan, A. N.; Oncken, M. P.; Maciel Filho, R.; Wolf Maciel, M. R. A roadmap for renewable $\mathrm{C} 2-\mathrm{C} 3$ glycols production: a process engineering approach. Green Chem. 2019, 21, 5168-5194.

(5) Ji, N.; Zhang, T.; Zheng, M.; Wang, A.; Wang, H.; Wang, X.; Chen, J. G. Direct catalytic conversion of cellulose into ethylene glycol using nickel-promoted tungsten carbide catalysts. Angew. Chem., Int. Ed. 2008, 47, 8510-8513.

(6) Zheng, M.; Pang, J.; Wang, A.; Zhang, T. One-pot catalytic conversion of cellulose to ethylene glycol and other chemicals: From fundamental discovery to potential commercialization. Chin. J. Catal. 2014, 35, 602-613.

(7) Zheng, M. Y.; Wang, A. Q.; Ji, N.; Pang, J. F.; Wang, X. D.; Zhang, T. Transition metal-tungsten bimetallic catalysts for the conversion of cellulose into ethylene glycol. ChemSusChem 2010, 3, 63-66.

(8) Li, N.; Ji, Z.; Wei, L.; Zheng, Y.; Shen, Q.; Ma, Q.; Tan, M.; Zhan, M.; Zhou, J. Effect of the surface acid sites of tungsten trioxide for highly selective hydrogenation of cellulose to ethylene glycol. Bioresour. Technol. 2018, 264, 58-65.

(9) Hamdy, M. S.; Eissa, M. A.; Keshk, S. M. A. S. New catalyst with multiple active sites for selective hydrogenolysis of cellulose to ethylene glycol. Green Chem. 2017, 19, 5144-5151.
(10) Zheng, M.; Pang, J.; Sun, R.; Wang, A.; Zhang, T. Selectivity Control for Cellulose to Diols: Dancing on Eggs. ACS Catal. 2017, 7, 1939-1954.

(11) Xu, G.; Wang, A.; Pang, J.; Zheng, M.; Yin, J.; Zhang, T. Remarkable effect of extremely dilute $\mathrm{H} 2 \mathrm{SO} 4$ on the cellulose conversion to ethylene glycol. Appl. Catal., A 2015, 502, 65-70.

(12) Wang, A.; Zhang, T. One-pot conversion of cellulose to ethylene glycol with multifunctional tungsten-based catalysts. Acc. Chem. Res. 2013, 46, 1377-1386.

(13) Zhao, G.; Zheng, M.; Sun, R.; Tai, Z.; Pang, J.; Wang, A.; Wang, X.; Zhang, T. Ethylene glycol production from glucose over W$\mathrm{Ru}$ catalysts: Maximizing yield by kinetic modeling and simulation. AIChE J. 2017, 63, 2072-2080.

(14) Tai, Z.; Zhang, J.; Wang, A.; Pang, J.; Zheng, M.; Zhang, T. Catalytic conversion of cellulose to ethylene glycol over a low-cost binary catalyst of Raney Ni and tungstic acid. ChemSusChem 2013, 6, $652-658$.

(15) Liao, Y.; de Beeck, B. O.; Thielemans, K.; Ennaert, T.; Snelders, J.; Dusselier, M.; Courtin, C. M.; Sels, B. F. The role of pretreatment in the catalytic valorization of cellulose. Mol. Catal. 2020, 487, No. 110883.

(16) Pang, J.; Zheng, M.; Wang, A.; Zhang, T. Catalytic Hydrogenation of Corn Stalk to Ethylene Glycol and 1,2-Propylene Glycol. Ind. Eng. Chem. Res. 2011, 50, 6601-6608.

(17) Pang, J.; Zheng, M.; Wang, A.; Sun, R.; Wang, H.; Jiang, Y.; Zhang, T. Catalytic conversion of concentrated miscanthus in water for ethylene glycol production. AIChE J. 2014, 60, 2254-2262.

(18) Fabičovicová, K.; Lucas, M.; Claus, P. From Barley Straw to Valuable Polyols: A Sustainable Process Using Ethanol/Water Mixtures and Hydrogenolysis over Ruthenium-Tungsten Catalyst. ChemSusChem 2016, 9, 2804-2815.

(19) Zhou, L.; Pang, J.; Wang, A.; Zhang, T. Catalytic conversion of Jerusalem artichoke stalk to ethylene glycol over a combined catalyst of WO3 and Raney Ni. Chin. J. Catal. 2013, 34, 2041-2046.

(20) Li, C.; Zheng, M.; Wang, A.; Zhang, T. One-pot catalytic hydrocracking of raw woody biomass into chemicals over supported carbide catalysts: simultaneous conversion of cellulose, hemicellulose and lignin. Energy Environ. Sci. 2012, 5, 6383-6390.

(21) Kumar, S.; Lange, J.-P.; Van Rossum, G.; Kersten, S. R. A. Liquefaction of Lignocellulose: Process Parameter Study To Minimize Heavy Ends. Ind. Eng. Chem. Res. 2014, 53, 11668-11676.

(22) Sluiter, A.; Hames, B.; Ruiz, R.; Scarlata, C.; Sluiter, J.; Templeton, D.; Crocker, D. Determination of Structural Carbohydrates and Lignin in Biomass, NREL/TP-510-42618; National Renewable Energy Laboratory, 2012.

(23) Sluiter, A.; Ruiz, R.; Scarlata, C.; Sluiter, J.; Templeton, D. Determination of Extractives in Biomass, NREL/TP-510-42619; National Renewable Energy Laboratory, 2008.

(24) Sluiter, A.; Hames, B.; Ruiz, R.; Scarlata, C.; Sluiter, J.; Templeton, D. Determination of Ash in Biomass, NREL/TP-51042622; National Renewable Energy Laboratory, 2008.

(25) Lange, J.-P. Catalysis for biorefineries-performance criteria for industrial operation. Catal. Sci. Technol. 2016, 6, 4759-4767.

(26) Zhang, J. Y.; Hou, B. L.; Wang, A. Q.; Li, Z. L.; Wang, H.; Zhang, T. Kinetic Study of the Competitive Hydrogenation of Glycolaldehyde and Glucose on $\mathrm{Ru} / \mathrm{C}$ with or Without AMT. AIChE J. 2015, 61, 224-238.

(27) Nimz, H. H.; Schmitt, U.; Schwab, E.; Wittmann, O.; Wolf, F. Wood; Wiley-VCH Verlag GmbH \& Co. KGaA: Weinheim, 2000; Vol. 39, pp 453-505.

(28) Vassilev, S. V.; Baxter, D.; Andersen, L. K.; Vassileva, C. G.; Morgan, T. J. An overview of the organic and inorganic phase composition of biomass. Fuel 2012, 94, 1-33. 